\title{
Similarities in the electrical properties of transition metal-hydrogen complexes in silicon
}

\author{
J.-U. Sachse ${ }^{a, *}$, E.Ö. Sveinbjörnsson ${ }^{b}$, N. Yarykin ${ }^{c}$, J. Weber ${ }^{\text {a }}$ \\ a Max-Planck-Institut für Festkörperforschung, D-70569 Stuttgart, Germany \\ ${ }^{\mathrm{b}}$ Department of Microelectronics ED, Chalmers University of Technology, S-41296 Göteborg, Sweden \\ ${ }^{\mathrm{c}}$ Institute of Microelectronics Technology RAS, 142432 Chernogolovka, Russia
}

\begin{abstract}
We review our recent studies on the reactions of hydrogen with transition-metals ( $\mathrm{Pd}, \mathrm{Pt}, \mathrm{Ag}$, and $\mathrm{Au}$ ) in crystalline $\mathrm{Si}$. Hydrogen was incorporated into the samples by wet-chemical etching. Deep-level transient spectroscopy (DLTS) on Schottky diodes reveals several transition metal-hydrogen complexes in $\mathrm{n}$ - and p-type samples. From DLTS profiling, we are able to estimate the number $i$ of hydrogen atoms in the $\mathrm{TM}-\mathrm{H}_{i}$ complexes. All complexes with $i=1,2$ are electrically active. Striking similarities are found for isoelectronic complexes, e.g. $\mathrm{Pt}-\mathrm{H}_{2}$ and $\mathrm{Au}-\mathrm{H}_{1}$. Transition metal complexes with more than three hydrogen atoms are likely to be electrically passive. All hydrogen related complexes disappear after heat treatments above $600 \mathrm{~K}$ for several hours. (C) 1999 Elsevier Science S.A. All rights reserved.
\end{abstract}

Keywords: Hydrogen; Transition metals; Silicon; Deep-level transient spectroscopy (DLTS)

\section{Introduction}

The interaction of hydrogen with transition metal impurities in silicon has drawn much attention in recent years. The first studies only reported on the electrical passivation of the isolated metal defects by hydrogenation [1,2]. Recently, the formation of novel electrically active metal-hydrogen complexes has been observed for a variety of transition metals by using deep-level transient spectroscopy (DLTS) [3-9]. In the latter case the assignment to hydrogen related defects is based on the appearance of new levels in the DLTS spectrum after sample hydrogenation and the corresponding defects are only detected at depths where hydrogen has been incorporated during processing. Although DLTS does usually not provide information on the microscopic defect structure we were able to estimate the number of $\mathrm{H}$ atoms in the various complexes by applying a simple model based on the concentration depth distributions of the corresponding defects [10]. This method together with detailed investigations of the

\footnotetext{
* Corresponding author. Present address: Siemens Microelectronics Center, D-01099 Dresden, Germany. Tel.: + 49-351-886-1752; fax: + 49-351-886-1753; e-mail: jens-uwe.sachse@hl.siemens.de.
}

thermal stabilities of the different centers give additional insight into the reactions which take place during complex formation and dissociation.

In this paper we concentrate our discussion on the interaction of hydrogen with transition metals with almost filled d-shells (Pt, Pd, Au, Ag). These metal impurities form isolated substitutional defects in silicon with electronic properties that are related to the lattice vacancy [11]. In the following we discuss how the close electronic relationship between the isolated metal defects transforms into relations between the various hydrogen related complexes.

\section{Experimental}

For our studies we used phosphorus or boron doped $\mathrm{FZ}$ or $\mathrm{CZ} \mathrm{Si}$ with shallow dopant concentrations in the range of $1 \times 10^{14}-1 \times 10^{16} \mathrm{~cm}^{-3}$. The transition metals were introduced either by evaporation of the metals on one side of an as received silicon wafer (FZ or $\mathrm{CZ}$ ) followed by heat treatment in a diffusion furnace between 800 and $1000^{\circ} \mathrm{C}$ or by introducing the metals into the silicon melt during the crystal growth [12]. The total concentration of the electrically active 
metal atoms varied in the samples and was strongly dependent on the particular metal. Typical concentrations were $5 \times 10^{13} \mathrm{~cm}^{-3}$ for the melt doped samples and $1 \times 10^{13}-1 \times 10^{15} \mathrm{~cm}^{-3}$ for the diffused samples.

Hydrogenation was performed by wet-chemical etching of the samples at room temperature in a 1:2:1 mixture of $\mathrm{HF}, \mathrm{HNO}_{3}$, and $\mathrm{CH}_{3} \mathrm{COOH}$ before evaporation of the Schottky contacts. The Schottky diodes were formed at room temperature by vacuum evaporation of aluminium for p-type samples and gold for n-type samples through a metal mask. An eutectic InGa alloy was rubbed onto the back side of the samples to facilitate an ohmic contact. In addition, cleaved samples were made by breaking the sample from the crystal rod and forming the contacts directly onto the sample surfaces without any additional surface treatment. The cleaved samples are virtually free from hydrogen and serve as a reference.

Wet-chemical etching incorporates hydrogen only in the region close to the sample surface [13] which can be detected by the formation of neutral hydrogen-acceptor and -donor pairs, respectively. Annealing under reverse bias is a convenient technique in p-type Si to inject hydrogen deeper into the bulk [14]. After an appropriate treatment the hydrogen is located at the end of the space charge region. In this case the depth profiles of the shallow boron concentration and the hydrogen related deep centers show an unambiguous correlation which supports the identification of the transition metal-hydrogen complexes. However, this method can not be applied in n-type Si for the intermediate shallow dopant concentrations used in this study.

Since the Schottky diodes deteriorated during annealing $>\approx 150^{\circ} \mathrm{C}$ the annealing studies at higher temperatures were made as follows: the samples were first etched, thereafter heat treated in a diffusion furnace in argon ambient and finally the Schottky contacts were prepared without any additional etching prior to the metallization.

A computerized DLTS system is used for detection of the deep levels. The capacitance transients are either directly measured with a transient recorder or integrated by a lock-in amplifier. During the DLTS concentration depth profiling the sample is held at a constant reverse bias, while the filling pulse height is varied [15]. The profiles are calculated taking into account the non-uniform shallow dopant profiles due to hydrogen passivation of the dopants. To increase the accuracy the measurements were repeated with different reverse biases resulting in an error in the determination of the deep level concentration of the order of $5 \%$ of the absolute concentration. The capture cross sections of majority carriers are extracted from the electron or hole capture rates which are determined by the filling pulse method [16]. The shallow dopant concentration profiles are determined from capacitance-voltage $(\mathrm{CV})$ measurements $(1 \mathrm{MHz})$.
By studying the electric field dependence of the emission rates and the temperature dependence of the capture cross sections of the particular levels we are able to determine their corresponding donor or acceptor character. Single deep donors in the lower half of the band gap and single deep acceptors in the upper half of the band gap do not show any field dependence of their emission rates as expected for centers not charged after carrier emission. In contrast, defects with donor states in the upper half of the band gap as well as defects with acceptor states in the lower half of the band gap remain charged after carrier emission and the Coulombic attraction between them and the emitted carriers leads to an enhancement in the emission rate with increasing electric fields. Carrier emission from possible double donor or acceptor states results in a repulsive interaction between the defect and the emitted carrier. Such centers exhibit a field dependence of the emission rate which is, however, weaker than in the first case $[17,18]$. A more characteristic feature of double donor and acceptor states is their small, but thermally activated capture cross section.

\section{Results}

The interaction of all four transition metals $(\mathrm{Pt}, \mathrm{Pd}$, $\mathrm{Au}, \mathrm{Ag}$ ) with hydrogen leads to the formation of various metal-hydrogen complexes which are either electrically active or passive [4,19-21]. This does not depend on the particular kind of metal doping applied. The appearance of new levels after hydrogenation indicating the formation of hydrogen related defects is always accompanied by reduction of the DLTS signals of the isolated metal defects. An example is given in Fig. 1 where the DLTS spectra of platinum doped n-type Si measured in a cleaved sample, i.e. before wet chemical etching (curve (a)), and after etching (curve (b)) are shown. Before hydrogenation we only detect the well-known acceptor level of isolated substitutional platinum. Etching results in a significant decrease in its intensity while two new peaks appear in the spectrum at 90 and $250 \mathrm{~K}$. The concentration depth profiles of both centers (not shown) exhibit a maximum in the region close to the sample surface, where hydrogen has been incorporated, and decrease rapidly towards the bulk.

Heat treatments between 300 and $500 \mathrm{~K}$ result in several transformations between the various metal-hydrogen complexes. After $1 \mathrm{~h}$ annealing at temperatures $>600 \mathrm{~K}$, however, the hydrogen related levels disappear and the signals of the isolated defects fully recover. We will discuss general features of the thermal transformation process in a later chapter.

The detection of new peaks in the DLTS spectrum after hydrogenation is a first indication of the formation of electrically active hydrogen-related complexes. 


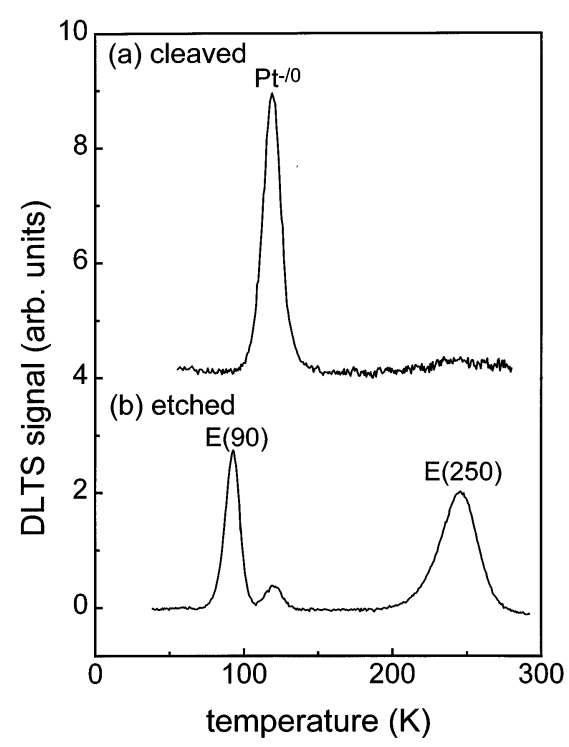

Fig. 1. Deep level transient spectroscopy spectra of Pt doped n-type $\mathrm{Si}$; (a) cleaved sample; (b) after etching.

Table 1 contains the electrical data of all hydrogen related levels for the transition metals studied. In the following we will concentrate on the identification of the different complexes by analyzing their concentration depth profiles. Particular problems occur when a complex is amphoteric and causes several levels in the band gap. Since their depth profiles should then be correlated all the profiles measured in $\mathrm{p}$ - and n-type $\mathrm{Si}$ have to be compared in an appropriate way. For such a comparison we first have to consider the different impact of the shallow dopants on the hydrogen distribution in the samples which affects the shape of the profiles of the deep defects. This is especially true at room temperature but can be avoided by a heat treatment at temperatures $>450 \mathrm{~K}$ when all $\mathrm{B}-\mathrm{H}-$ and $\mathrm{P}-\mathrm{H}$-pairs are dissociated. Furthermore, the different shallow donor and acceptor concentrations in n- and p-type samples are accounted for by using the depth profile of the isolated defects as a reference, i.e. the profiles of the respective donor level in p-type $\mathrm{Si}$ and the respective acceptor level in n-type Si must coincide.

Fig. 2 shows the depth profiles of all platinum related levels after etching and a subsequent $1 \mathrm{~h}$ heat treatment at $470 \mathrm{~K}$. Relative concentration values which are related to the initial $\mathrm{Pt}$ concentration in the cleaved samples are used to take into consideration the differences in the absolute Pt concentrations in n- and p-type $\mathrm{Si}$, respectively. All defects show an inhomogenuous distribution. We find a remarkable coincidence in the profiles of the levels $\mathrm{E}(90)$ in n-type and $\mathrm{H}(210)$ in p-type Si. This is also true after annealing studies at other temperatures $>450 \mathrm{~K}$ and indicates that both levels belong to the same complex. The other two levels $\mathrm{E}(250)$ in n-type and $\mathrm{H}(150)$ in p-type $\mathrm{Si}$ show no similarities in their concentration profiles and are therefore assigned to different $\mathrm{Pt}-\mathrm{H}$ defects. Furthermore, we note that the total concentration of electrically

Table 1

Energy levels determined from Arrhenius plots of the emission rates, capture cross sections $\sigma_{\mathrm{n}, \mathrm{p}}$ determined by variation of the filling pulse length and level character ${ }^{\mathrm{a}}$

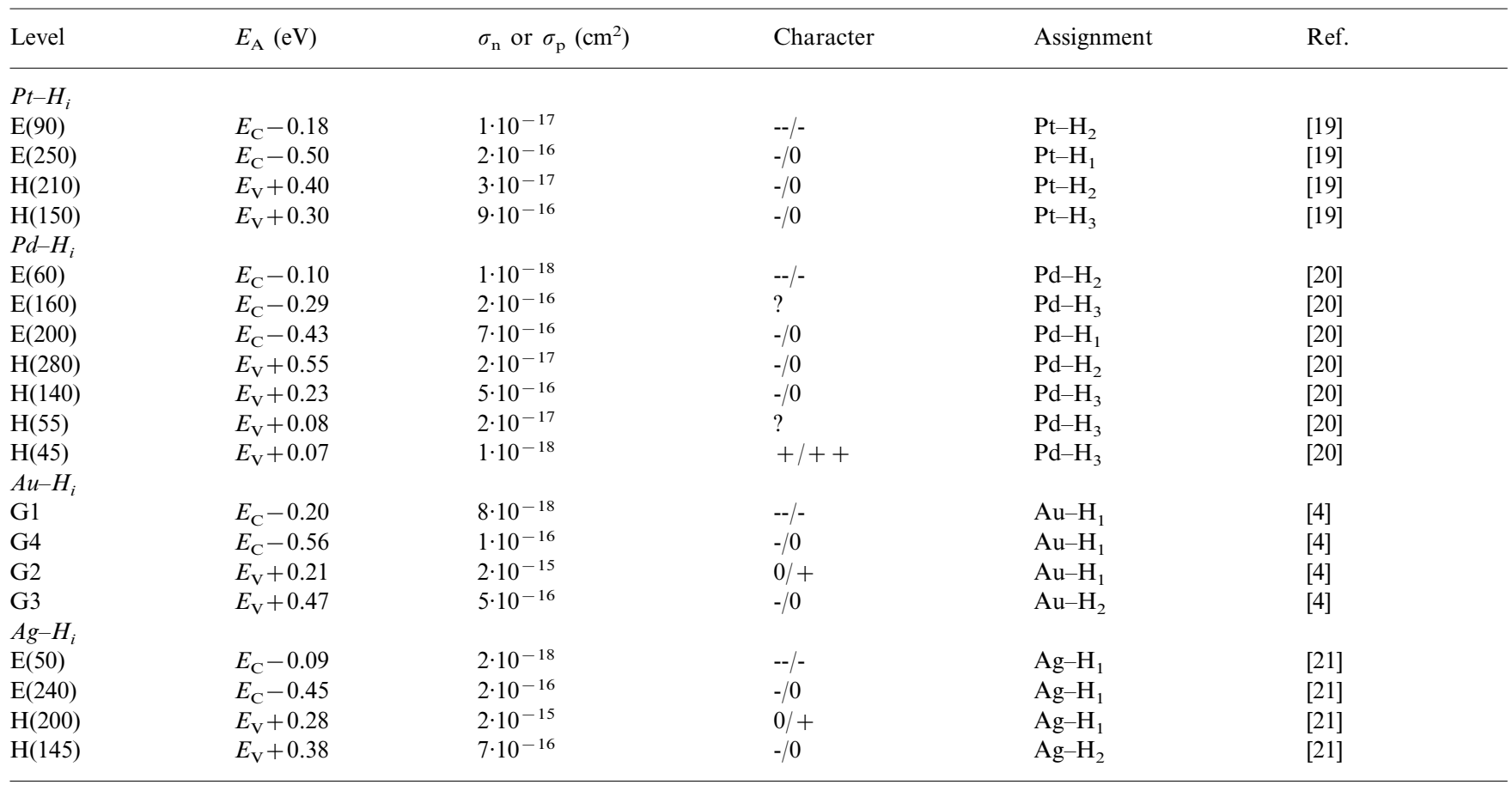

${ }^{a}$ Assignment of the deep levels is based on the analysis of the depth profiles. 


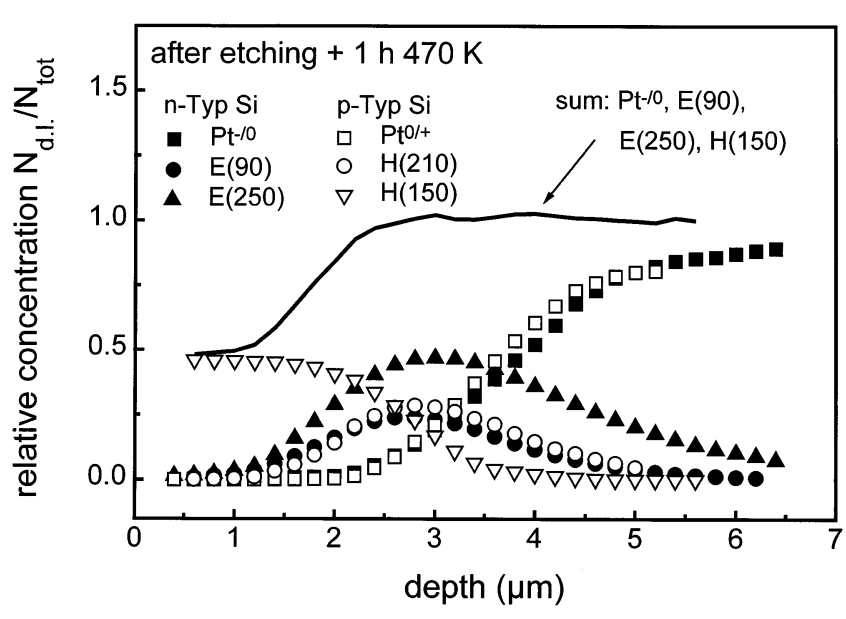

Fig. 2. Depth profiles of the platinum-related defects in Si after etching and subsequent annealing at $470 \mathrm{~K}$ for $1 \mathrm{~h}$, related to the initial Pt concentration in the cleaved samples.

active platinum (isolated or involved in complexes) is uniform and agrees with the initial Pt concentration except for a region very close to the surface $(<2 \mu \mathrm{m})$ where the total concentration is reduced by $\approx 50 \%$. This decrease is most probably due to the formation of an additional electrically inactive $\mathrm{Pt}-\mathrm{H}$ complex.

A semilogarithmic plot as shown in Fig. 3 reveals that the profiles of hydrogen related defects decrease exponentially with the depth towards the bulk. This behavior can be explained by a model which describes the complex formation as a subsequent addition of hydrogen atoms to the isolated metal impurity TM. Feklisova and Yarykin showed that under these conditions the concentration of a complex $\left[\mathrm{TM}-\mathrm{H}_{i}\right]$ with $i$ hydrogen atoms can be described in a quasistationary solution by an exponential decrease at greater depths $x$ [10]:

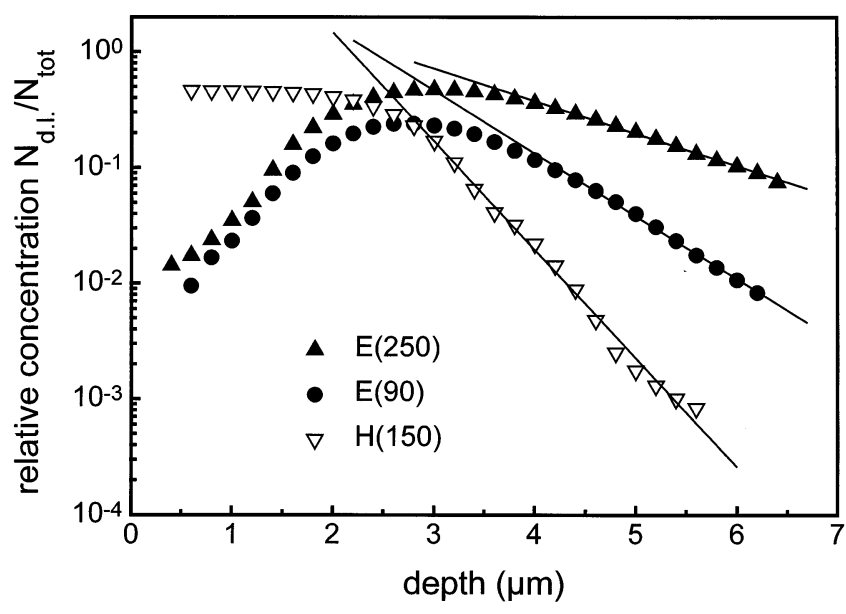

Fig. 3. Depth profiles of the Pt-H-related levels E(250), E(90), and $\mathrm{H}(210)$ after etching and subsequent annealing at $470 \mathrm{~K}$ for $1 \mathrm{~h}$. The solid lines represent fits using Eq. (1) and provide the corresponding penetration depths $L_{i}$.
$\left[\mathrm{TM}-\mathrm{H}_{i}\right] \sim \exp \left(-x / L_{i}\right)$

Furthermore, the characteristic penetration depth $L_{i}$ is inversely proportional to $i$. Thus the ratios of the penetration depths obtained by fitting the slopes of the concentration depth profiles with Eq. (1) can be formed for the various complexes and provide an estimate of the number of $\mathrm{H}$ atoms.

This means for platinum (Fig. 3) that $\mathrm{E}(250)$ has the largest penetration depth and is therefore assigned to a complex with only one hydrogen atom. E(90) and $\mathrm{H}(210)$ exhibit penetration depths of about half of $\mathrm{E}(250)$ and are different levels of a complex with two $\mathrm{H}$ atoms. An amphoteric $\mathrm{Pt}-\mathrm{H}_{2}$ complex with similar electrical properties has also been detected by Uftring et al. [22] using electron paramagnetic resonance and vibrational spectroscopy. Calculations of Resende confirm this assignment [23]. We further assign $\mathrm{H}(150)$ to $\mathrm{Pt}-\mathrm{H}_{3}$ although the fit of the profile becomes more difficult with increasing number of $\mathrm{H}$ atoms.

We applied this procedure to all the hydrogen-related centers for all the metals studied. The corresponding assignments are included in Table 1. For each metal we found one complex with at least two different levels. The trivalent behavior of $\mathrm{Au}-\mathrm{H}$ was already reported earlier by Sveinbjörnsson and Engström [4], a similar defect is observed for silver. The hydrogenation of palladium doped Si results in the formation of a bivalent $\mathrm{Pd}-\mathrm{H}_{2}$ complex similar to $\mathrm{Pt}-\mathrm{H}_{2}$. Furthermore, $\mathrm{H}(45)$ and $\mathrm{H}(55)$ are levels of the same complex containing three $\mathrm{H}$ atoms while $\mathrm{H}(140)$ and $\mathrm{E}(160)$ most probably belong to different complexes with three $\mathrm{H}$ atoms due to differences in the annealing behavior [24].

In addition to the formation of electrically active complexes, each metal impurity can also be passivated completely. The depth profiles of the corresponding passive complexes are quite uncertain because of their indirect determination via the profiles of all other defects. A characteristic feature is, however, their presence only in the hydrogen-richest regions suggesting that they contain more $\mathrm{H}$ atoms than all the other electrically active complexes. A rough estimate gives that the passive configuration for $\mathrm{Pt}$ and $\mathrm{Pd}$ contains four or more $\mathrm{H}$ atoms while in the case of $\mathrm{Au}$ and $\mathrm{Ag}$ only three atoms are needed for complete passivation.

\section{Discussion}

\subsection{Formation and thermal stability of transition metal-hydrogen complexes}

We observe for all four transition metals significant differences in the formation of hydrogen related defects in $\mathrm{n}$ - and p-type $\mathrm{Si}$, respectively. In n-type material the complexes are already observed after etching while an 
(a) Pd, Au, Ag

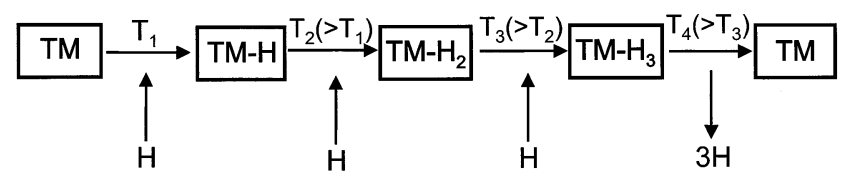

(b) $\mathrm{Pt}$

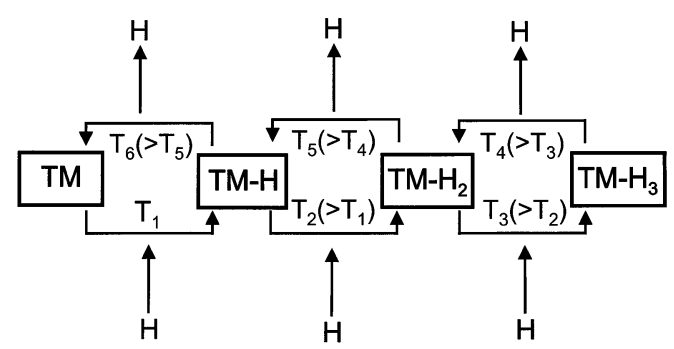

Fig. 4. Scheme of the formation and dissociation reactions for transition metal-hydrogen complexes $\left(\mathrm{TM}-\mathrm{H}_{i}\right)$. The number of $\mathrm{H}$ atoms in the hydrogen-richest complex can differ for the different metals.

additional annealing step is needed in p-type $\mathrm{Si}$, usually 30 min at $\approx 370 \mathrm{~K}$. These differences can be explained by the competition between shallow dopants and metal impurities for free hydrogen.

In p-type $\mathrm{Si}$ the boron acceptors are negatively charged at room temperature while the deep metal impurities are mostly positively charged. Since hydrogen exists as $\mathrm{H}^{+}$ion the formation of boron-hydrogen pairs is strongly favored due to Coulombic attraction. An increase in temperature results in the dissociation of the $\mathrm{B}-\mathrm{H}$ pairs and the release of hydrogen. At the same time the metal defects become electrically neutral due to the shift of the Fermi energy and can now react with hydrogen without Coulombic repulsion. In contrast, pairing of positively charged phosphorus donors and hydrogen which is neutral or positively charged is less efficient in n-type Si. Thus a considerable portion of hydrogen can complex with the negatively charged or neutral metals already at room temperature.

Taking into account these differences in $\mathrm{n}$ - and $\mathrm{p}$ type $\mathrm{Si}$ a more general picture of the formation of metal-hydrogen complexes can be drawn as illustrated in Fig. 4. The characteristic feature is that the transformation in hydrogen-rich complexes proceeds via the successive addition of $\mathrm{H}$ atoms although not all the steps can be detected directly. The scheme also includes the experimental fact that the complexes dissociate at higher temperatures. We find that the metals studied can be separated into two groups. In the case of $\mathrm{Pd}$, $\mathrm{Au}$, and $\mathrm{Ag}$ the complex with most $\mathrm{H}$ atoms is the most stable one and dissociates directly into the isolated metal defect and hydrogen. In contrast, for Pt we also observe a partial dissociation of complexes containing many $\mathrm{H}$ atoms with the result that the $\mathrm{Pt}-\mathrm{H}$ pair is the most stable defect. The reactivation of isolated defects takes place at $600 \mathrm{~K}$, except for gold where annealings at only $470-500 \mathrm{~K}$ are needed. The reasons for the different behavior are not clear yet, but are probably related to the particular microscopic structure.

\subsection{Electrical activity}

The electrical activity of the various hydrogen related complexes can partly be derived from the properties of the isolated substitutional defects. In the so-called vacancy model [11] platinum as 5d-transition metal and palladium as 4d-transition metal form substitutional defects and behave as isoelectronic relatives. This results in very similar electronic properties which have been experimentally verified. The same holds true for gold and silver.

The hydrogen related complexes with one or two $\mathrm{H}$ atoms are electrically active for all four metals and their energy levels are summarized in Fig. 5. We find a remarkable coincidence not only in the charge states in which the complexes occur, but also in the corresponding energy levels and capture cross sections (see also Table 1). This indicates that complexes of isoelectronic metals having the same number of hydrogen atoms do not strongly depend on the specific nature of the metal. As in the case of isolated defects this gives evidence that the interaction with hydrogen is also controlled by the behavior of the vacancy.

The influence of the number of $\mathrm{H}$ atoms on the electrical activity is visualized in Fig. 6. A strong shift of the single $\mathrm{Pt}$ acceptor level is found with the addition of $\mathrm{H}$ atoms. It can be extended under the assumption that the passive complex contains four $\mathrm{H}$ atoms. This would mean that the acceptor level is shifted below the valence band edge. The same trend is also observed for
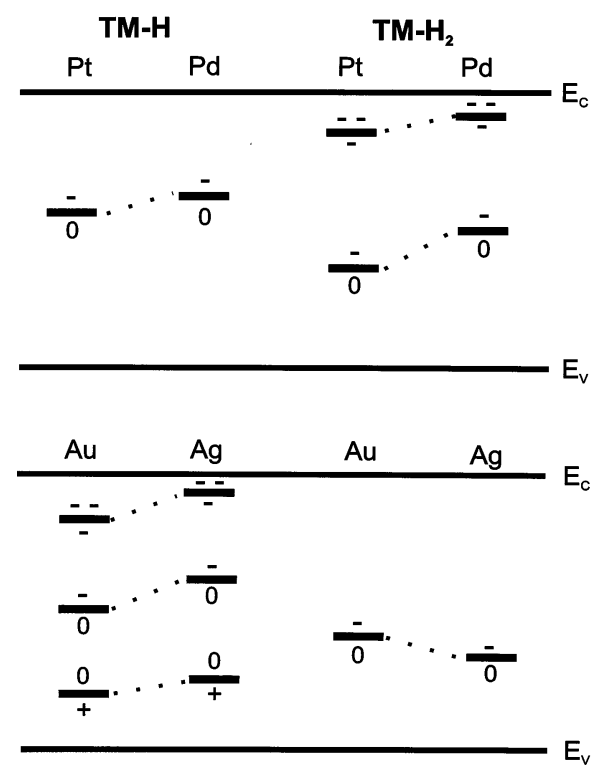

Fig. 5. Energy-level diagram showing the levels of transition metalhydrogen related complexes containing one or two $\mathrm{H}$ atoms. 


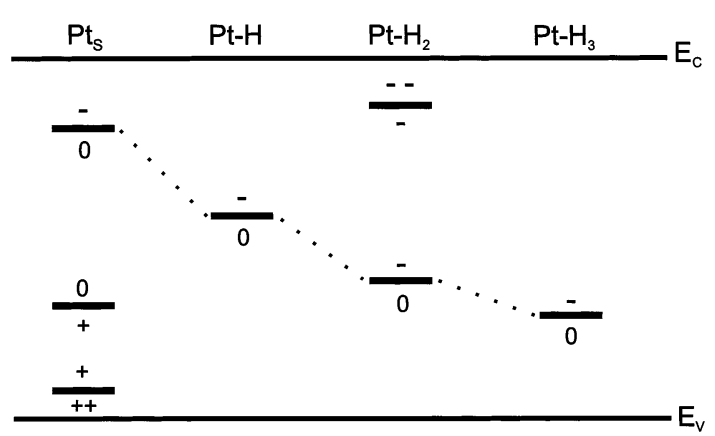

Fig. 6. Energy-level diagram for platinum and platinum-hydrogen related defects. The dashed line indicates a shift of the single acceptor towards the valence band edge with an increasing number of $\mathrm{H}$ atoms.

Pd, but not for gold and silver. The shift towards the valence band may be explained within the framework of the vacancy model by the assumption that each $\mathrm{H}$ atom adds an electron into the $t_{2}$-level of the vacancy, which leads to a decrease of the corresponding energy level.

Furthermore, we note correlations in the electrical activity between isoelectronic complexes of metals with different numbers of valence electrons like platinum and gold. Fig. 7 shows that $\mathrm{Pt}-\mathrm{H}_{2}$ and $\mathrm{Au}-\mathrm{H}$ exhibit similarities in the two acceptor levels; a donor level is not observed for $\mathrm{Pt}-\mathrm{H}_{2}$ and is probably located below the valence band edge. Adding one more $\mathrm{H}$ atom results in the formation of $\mathrm{Pt}-\mathrm{H}_{3}$ and $\mathrm{Au}-\mathrm{H}_{2}$ and their acceptor levels almost coincide.

An explanation of these similarities is again possible by the correlation of the substitutional defects of both transition metals with the vacancy. Neutral isolated gold contains one more valence electron than platinum. Assuming that the process of complexing can be understood in the microscopic picture in terms of simply adding electrons to the defect, the complexes compared above contain the same number of valence electrons [24]. This means that the $t_{2}$-level would contain four electrons for $\mathrm{Pt}-\mathrm{H}_{2}$ and $\mathrm{Au}-\mathrm{H}$ and five electrons for $\mathrm{Au}-\mathrm{H}_{2}$ and $\mathrm{Pt}-\mathrm{H}_{3}$. The addition of another electron leads to a complete filling of the threefold degenerate

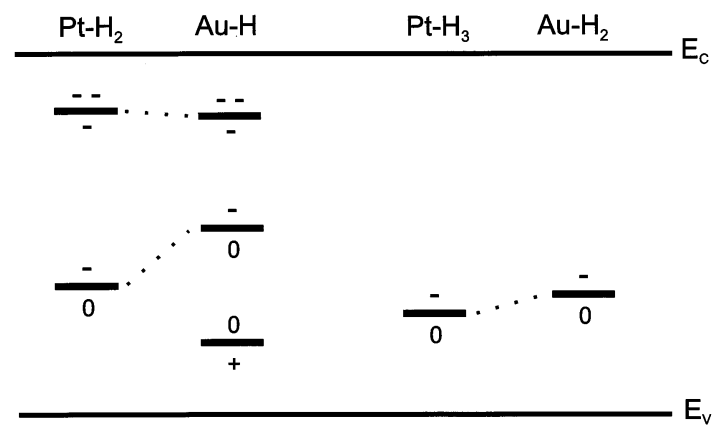

Fig. 7. Energy-level diagram of isoelectronic hydrogen-related complexes for platinum and gold. $t_{2}$-level which corresponds to a complete passivation of the particular defect. Our experimental studies show that the passive complexes of $\mathrm{Au}$ and $\mathrm{Pt}$ indeed may contain three and four $\mathrm{H}$ atoms, respectively, which would support the basic ideas described above. However, a more sophisticated analysis is necessary to understand the effect of hydrogen on the electronic structure of the transition metal-hydrogen system.

\section{Acknowledgements}

We thank H.-J. Queisser for his steady support throughout this work. We are indebted to G. I. Andersson for providing the computer program for the depth profile calculations, to $\mathrm{H}$. Lemke for providing the samples doped during crystal growth and to A. Resende and R. Jones for communicating theoretical results to us prior to publications. The excellent technical assistance of W. Heinz and W. Krause is acknowledged.

\section{References}

[1] S.J. Pearton, E.E. Haller, J. Appl. Phys. 54 (1983) 3613.

[2] S.J. Pearton, in: J.I. Pankove, N.M. Johnson (Eds.), Semiconductors and Semimetals, Academic, San Diego, CA, 1991, p. 65.

[3] E.Ö. Sveinbjörnsson, O. Engström, Appl. Phys. Lett. 61 (1992) 2323.

[4] E.Ö Sveinbjörnsson, O. Engström, Phys. Rev. B52 (1995) 4884.

[5] T. Sadoh, H. Nakashima, T. Tsurushima, J. Appl. Phys. 72 (1992) 520.

[6] T. Sadoh, M. Watanabe, H. Nakashima, T. Tsurushima, J. Appl. Phys. 75 (1994) 3978.

[7] H. Feichtinger, E. Sturm, Mater. Sci. Forum 143-147 (1994) 111.

[8] W. Jost, J. Weber, Phys. Rev. B54 (1996) R11038.

[9] W. Jost, J. Weber, H. Lemke, Semicond. Sci. Technol. 11 (1996) 22.

[10] O.V. Feklisova, N.A. Yarykin, Semicond. Sci. Technol. 12 (1997) 742.

[11] G.D. Watkins, Physica 117B-118B (1983) 9.

[12] H. Lemke, in: H.R. Huff, W. Bergholz, K. Sumino (Eds.), Semiconductor Silicon, The Electrochemical Society, Pennington, NJ, 1994, p. 695.

[13] A.J. Tavendale, A.A. Williams, S.J. Pearton, in: M. Stavola, S.J. Pearton, G. Davies (Eds.), Defects in Electronic Materials, MRS Symposia Proceedings No. 104, Materials Research Society, Pittsburgh, PA, 1988, p. 285.

[14] T. Zundel, J. Weber, Phys. Rev B39 (1989) 13549.

[15] H.G. Grimmeiss, E. Janzén, B. Skarstam, A. Lodding, J. Appl. Phys. 51 (1980) 6238.

[16] D.V. Lang, J. Appl. Phys. 45 (1974) 3023.

[17] H. Zimmermann, H. Ryssel, Appl. Phys. Lett. 58 (1991) 499.

[18] J.-U. Sachse, W. Jost, J. Weber, H. Lemke, Appl. Phys. Lett. 71 (1997) 1379.

[19] J.-U. Sachse, E.Ö. Sveinbjörnsson, W. Jost, J. Weber, H. Lemke, Phys. Rev. B55 (1997) 16176.

[20] J.-U. Sachse, J. Weber, H. Lemke, Mater. Sci. Forum 258-263 (1997) 307. 
[21] N. Yarykin, J.-U. Sachse, J. Weber, H. Lemke, Mater. Sci. Forum 258-263 (1997) 301.

[22] S.J. Uftring, M. Stavola, P.M. Williams, G.D. Watkins, Phys. Rev. B51 (1995) 9612.
[23] A. Resende, private communication.

[24] J.-U. Sachse, PhD Thesis University of Stuttgart, 1997, submitted for publication. 